\title{
Existence and exponential stability of an equilibrium point for fuzzy BAM neural networks with time-varying delays in leakage terms on time scales
}

\author{
Yongkun Li*, Li Yang and Lijie Sun
}

\section{"Correspondence: yklie@ynu.edu.cn Department of Mathematics, Yunnan University, Kunming, Yunnan 650091, People's Republic of China}

\begin{abstract}
In this paper, by using a fixed point theorem and differential inequality techniques, we consider the existence and global exponential stability of an equilibrium point for a class of fuzzy bidirectional associative memory neural networks with time-varying delays in leakage terms on time scales. We also present a numerical example to show the feasibility of obtained results. The results of this paper are completely new and complementary to the previously known results.
\end{abstract}

MSC: 34B37; 34N05

Keywords: fuzzy BAM neural networks; exponential stability; leakage terms; time scales

\section{Introduction}

The bidirectional associative memory (BAM) neural networks were first introduced by Kosto in 1988 [1]. These are special recurrent neural networks that can store bipolar vector pairs and are composed of neurons arranged in two layers. The neurons in one layer are fully interconnected to the neurons in the other layer, while there are no interconnections among neurons in the same layer.

In recent years, due to their wide range of applications, for example, pattern recognition, associative memory, and combinatorial optimization, BAM neural networks have received much attention. There are lots of results on the existence and stability of an equilibrium point, periodic solutions or almost periodic solutions of BAM neural networks [2-10].

Based on traditional cellular neural networks, Yang and Yang proposed a fuzzy cellular neural network, which integrates fuzzy logic into the structure of traditional cellular neural networks and maintains local connectedness among cells [11]. The fuzzy neural network has fuzzy logic between its template input and/or output besides the sum of product operation. Studies have revealed that the fuzzy neural network is very useful for image processing problems, which is a cornerstone in image processing and pattern recognition. Besides, in reality, time delays often occur due to finite switching speeds of the amplifiers and communication time and can destroy a stable network or cause sustained oscillations, bifurcation or chaos. Hence, it is important to consider both the fuzzy logic and delay effect on dynamical behaviors of neural networks. There have been many results on the

(c) $2013 \mathrm{Li}$ et al.: licensee Springer. This is an Open Access article distributed under the terms of the Creative Commons Attribution License (http://creativecommons.org/licenses/by/2.0), which permits unrestricted use, distribution, and reproduction in any medium, provided the original work is properly cited. 
fuzzy neural networks with time delays [12-17]. Moreover, time delay in the leakage term also has a great impact on the dynamics of neural networks. As pointed out by the author in [18], time delay in the stabilizing negative feedback term has a tendency to destabilize a system. Therefore, it is meaningful to consider fuzzy neural networks with time delays in the leakage terms [19-24].

In fact, both continuous and discrete systems are very important in implementation and applications. To avoid the trouble of studying the dynamical properties for continuous and discrete systems respectively, it is meaningful to study those on time scales, which was initiated by Stefan Hilger in his $\mathrm{PhD}$ thesis, in order to unify continuous and discrete analyses. Lots of scholars have studied neural networks on time scales and obtained many good results [25-34]. However, to the best of our knowledge, there is no paper published on the stability of fuzzy BAM neural networks with time delays in the leakage terms on time scales.

Motivated by the above, in this paper, we integrate fuzzy operations into BAM neural networks with time delays in the leakage terms and study the stability of considered neural networks on time scales. By using a fixed point theorem and differential inequality techniques, we consider the existence and global exponential stability of an equilibrium point for the following BAM neural network with time-varying delays in leakage terms on time scales:

$$
\left\{\begin{aligned}
x_{i}^{\Delta}(t)= & -a_{i} x_{i}\left(t-\delta_{i}(t)\right)+\sum_{j=1}^{m} c_{j j} f_{j}\left(y_{j}\left(t-\tau_{j i}(t)\right)\right)+\bigwedge_{j=1}^{m} \alpha_{j i} f_{j}\left(y_{j}\left(t-\tau_{j i}(t)\right)\right) \\
& +\bigwedge_{j=1}^{m} T_{j i} \mu_{j}+\bigvee_{j=1}^{m} \beta_{j i} f_{j}\left(y_{j}\left(t-\tau_{j i}(t)\right)\right) \\
& +\bigvee_{j=1}^{m} H_{j i} \mu_{j}+I_{i}, \quad t \in \mathbb{T}, i=1,2, \ldots, n, \\
y_{j}^{\Delta}(t)= & -b_{j} y_{j}\left(t-\eta_{j}(t)\right)+\sum_{i=1}^{n} d_{i i} g_{i}\left(x_{i}\left(t-\sigma_{i j}(t)\right)\right)+\bigwedge_{i=1}^{n} p_{i j} g_{i}\left(x_{i}\left(t-\sigma_{i j}(t)\right)\right) \\
& +\bigwedge_{i=1}^{n} F_{i j} v_{i}+\bigvee_{i=1}^{n} q_{i j} g_{i}\left(x_{i}\left(t-\sigma_{i j}(t)\right)\right) \\
& +\bigvee_{i=1}^{n} G_{i j} v_{i}+J_{j}, \quad t \in \mathbb{T}, j=1,2, \ldots, m,
\end{aligned}\right.
$$

where $\mathbb{T}$ is a time scale; $n, m$ are the number of neurons in layers; $x_{i}(t)$ and $y_{j}(t)$ denote the activations of the $i$ th neuron and the $j$ th neuron at time $t ; a_{i}>0$ and $b_{j}>0$ represent the rate at which the $i$ th neuron and the $j$ th neuron will reset their potential to the resting state in isolation when they are disconnected from the network and the external inputs; $0<\delta_{i}<\delta_{i}^{+}$and $0<\eta_{j}<\eta_{j}^{+}$denote the leakage delays; $f_{j}, g_{i}$ are the input-output functions (the activation functions); $0 \leq \tau_{j i}(t) \leq \tau_{j i}$ and $0 \leq \sigma_{i j}(t) \leq \sigma_{i j}$ are transmission delays; $t-$ $\tau_{j i}(t) \in \mathbb{T}, t-\sigma_{i j}(t) \in \mathbb{T}, t-\delta_{i}(t) \in[0, \infty)_{\mathbb{T}}$ and $t-\eta_{j}(t) \in[0, \infty)_{\mathbb{T}} ; c_{j i}, d_{i j}$ are elements of feedback templates; $\alpha_{j i}, p_{i j}$ denote the elements of fuzzy feedback MIN templates and $\beta_{j i}$, $q_{i j}$ are the elements of fuzzy feedback MAX templates; $T_{j i}, F_{i j}$ are fuzzy feed-forward MIN templates and $H_{j i}, G_{i j}$ are fuzzy feed-forward MAX templates; $\mu_{j}, v_{i}$ denote the input of the $i$ th neuron and the $j$ th neuron; $I_{i}, J_{j}$ denote biases of the $i$ th neuron and the $j$ th neuron, $i=1,2, \ldots, n, j=1,2, \ldots, m, \wedge$ and $\bigvee$ denote the fuzzy AND and fuzzy OR operations, respectively.

The initial condition of (1.1) is of the form

$$
\begin{cases}x_{i}(s)=\varphi_{i}(s), & s \in[-\sigma, 0]_{\mathbb{T}}, \sigma=\max \left\{\max _{(i, j)} \sigma_{i j}, \max _{i} \delta_{i}^{+}\right\}, i=1,2, \ldots, n, \\ y_{j}(s)=\psi_{j}(s), & s \in[-\tau, 0]_{\mathbb{T}}, \tau=\max \left\{\max _{(i, j)} \tau_{j i}, \max _{j} \eta_{j}^{+}\right\}, j=1,2, \ldots, m,\end{cases}
$$

where $\varphi_{i}(\cdot), \psi_{j}(\cdot)$ denote positive real-valued continuous functions on $[-\sigma, 0]_{\mathbb{T}}$ and $[-\tau, 0]_{\mathbb{T}}$, respectively. 
For the sake of convenience, we introduce some notations. For matrix $D, D^{T}$ denotes the transpose of $D, \rho(D)$ denotes the spectral radius of $D$. A matrix or a vector $D \geq 0$ means that all entries of $D$ are greater than or equal to zero, $D>0$ can be defined similarly. For matrices or vectors $D$ and $E, D \geq E$ (respectively $D>E$ ) means that $D-E \geq 0$ (respectively $D-E>0)$.

Throughout this paper, we assume that the following condition holds:

$(\mathrm{H}) f_{j}, g_{i} \in C(\mathbb{R}, \mathbb{R})$ and there exist positive constants $L_{j}^{f}, L_{i}^{g}$ such that

$$
\left|f_{j}(u)-f_{j}(v)\right| \leq L_{j}^{f}|u-v|, \quad\left|g_{i}(u)-g_{i}(v)\right| \leq L_{i}^{g}|u-v|
$$

for all $u, v \in \mathbb{R}, i=1,2, \ldots, n, j=1,2, \ldots, m$.

The organization of the rest of this paper is as follows. In Section 2, we introduce some preliminary results which are needed in the later sections. In Section 3, we establish some sufficient conditions for the existence and uniqueness of the equilibrium point of (1.1). In Section 4, we prove the equilibrium point of (1.1) is globally exponentially stable. In Section 5, we give an example to illustrate the feasibility and effectiveness of our results obtained in previous sections.

\section{Preliminaries}

In this section, we state some preliminary results.

Definition 2.1 [25] Let $\mathbb{T}$ be a nonempty closed subset (time scale) of $\mathbb{R}$. The forward and backward jump operators $\sigma, \rho: \mathbb{T} \rightarrow \mathbb{T}$ and the graininess $\mu: \mathbb{T} \rightarrow \mathbb{R}_{+}$are defined, respectively, by

$$
\sigma(t)=\inf \{s \in \mathbb{T}: s>t\}, \quad \rho(t)=\sup \{s \in \mathbb{T}: s<t\} \quad \text { and } \quad \mu(t)=\sigma(t)-t .
$$

Lemma 2.1 [25] Assume that $p, q: \mathbb{T} \rightarrow \mathbb{R}$ are two regressive functions, then

(i) $e_{0}(t, s) \equiv 1$ and $e_{p}(t, t) \equiv 1$;

(ii) $e_{p}(t, s)=\frac{1}{e_{p}(s, t)}=e_{\ominus p}(s, t)$;

(iii) $e_{p}(t, s) e_{p}(s, r)=e_{p}(t, r)$;

(iv) $\left(e_{p}(t, s)\right)^{\Delta}=p(t) e_{p}(t, s)$.

Lemma 2.2 [25] Let $f$, $g$ be $\Delta$-differentiable functions on $T$, then

(i) $\left(v_{1} f+v_{2} g\right)^{\Delta}=v_{1} f^{\Delta}+v_{2} g^{\Delta}$ for any constants $v_{1}, v_{2}$;

(ii) $(f g)^{\Delta}(t)=f^{\Delta}(t) g(t)+f(\sigma(t)) g^{\Delta}(t)=f(t) g^{\Delta}(t)+f^{\Delta}(t) g(\sigma(t))$.

Lemma 2.3 [35] Assume that $p(t) \geq 0$ for $t \geq s$, then $e_{p}(t, s) \geq 1$.

Definition 2.2 [35] A function $r: \mathbb{T} \rightarrow \mathbb{R}$ is called regressive if

$$
1+\mu(t) r(t) \neq 0
$$

for all $t \in \mathbb{T}^{k}$. The set of all regressive and rd-continuous functions $r: \mathbb{T} \rightarrow \mathbb{R}$ will be denoted by $\mathcal{R}$. We define the set $\mathcal{R}^{+}=\{r \in \mathcal{R}: 1+\mu(t) r(t)>0, \forall t \in \mathbb{T}\}$.

Lemma 2.4 [35] Suppose that $p \in \mathcal{R}^{+}$, then 
(i) $e_{p}(t, s)>0$ for all $t, s \in \mathbb{T}$;

(ii) if $p(t) \leq q(t)$ for all $t \geq s, t, s \in \mathbb{T}$, then $e_{p}(t, s) \leq e_{q}(t, s)$ for all $t \geq s$.

Lemma 2.5 [35] If $p \in \mathcal{R}$ and $a, b, c \in \mathbb{T}$, then

$$
\left[e_{p}(c, \cdot)\right]^{\Delta}=-p\left[e_{p}(c, \cdot)\right]^{\sigma}
$$

and

$$
\int_{a}^{b} p(t) e_{p}(c, \sigma(t)) \Delta t=e_{p}(c, a)-e_{p}(c, b)
$$

Lemma 2.6 [35] Let $a \in \mathbb{T}^{k}, b \in \mathbb{T}$ and assume that $f: \mathbb{T} \times \mathbb{T}^{k} \rightarrow \mathbb{R}$ is continuous at $(t, t)$, where $t \in \mathbb{T}^{k}$ with $t>a$. Also assume that $f^{\Delta}(t, \cdot)$ is $r d$-continuous on $[a, \sigma(t)]$. Suppose that for each $\varepsilon>0$, there exists a neighborhood $U$ of $\tau \in[a, \sigma(t)]$ such that

$$
\left|f(\sigma(t), \tau)-f(s, \tau)-f^{\Delta}(t, \tau)(\sigma(t)-s)\right| \leq \varepsilon|\sigma(t)-s|, \quad \forall s \in U,
$$

where $f^{\Delta}$ denotes the derivative off with respect to the first variable. Then

(i) $g(t):=\int_{a}^{t} f(t, \tau) \Delta \tau$ implies $g^{\Delta}(t):=\int_{a}^{t} f^{\Delta}(t, \tau) \Delta \tau+f(\sigma(t), t)$;

(ii) $h(t):=\int_{t}^{b} f(t, \tau) \Delta \tau$ implies $h^{\Delta}(t):=\int_{t}^{b} f^{\Delta}(t, \tau) \Delta \tau-f(\sigma(t), t)$.

Definition 2.3 A point $z^{*}=\left(x_{1}^{*}, x_{2}^{*}, \ldots, x_{n}^{*}, y_{1}^{*}, y_{2}^{*}, \ldots, y_{m}^{*}\right)^{T} \in R^{n+m}$ is said to be an equilibrium point of (1.1) if $z(t)=z^{*}$ is a solution of (1.1).

Lemma 2.7 [17] Let $f_{j}$ be defined on $R, j=1,2, \ldots, m$. Then, for any $a_{i j} \in R, i=1,2, \ldots, n$, $j=1,2, \ldots, m$, we have the following estimations:

$$
\left|\bigwedge_{j=1}^{m} a_{i j} f_{j}\left(u_{j}\right)-\bigwedge_{j=1}^{m} a_{i j} f_{j}\left(v_{j}\right)\right| \leq \sum_{j=1}^{m}\left|a_{i j}\right|\left|f_{j}\left(u_{j}\right)-f_{j}\left(v_{j}\right)\right|
$$

and

$$
\left|\bigvee_{j=1}^{m} a_{i j} f_{j}\left(u_{j}\right)-\bigvee_{j=1}^{m} a_{i j} f_{j}\left(v_{j}\right)\right| \leq \sum_{j=1}^{m}\left|a_{i j}\right|\left|f_{j}\left(u_{j}\right)-f_{j}\left(v_{j}\right)\right|,
$$

where $u_{j}, v_{j} \in R, j=1,2, \ldots, m$.

Definition 2.4 [36] A real matrix $A=\left(a_{i j}\right)_{n \times n}$ is said to be an $M$-matrix if $a_{i j} \leq 0, i, j=$ $1,2, \ldots, n, i \neq j$ and all successive principal minors of $A$ are positive.

Lemma 2.8 [36] Let $A=\left(a_{i j}\right)_{n \times n}$ be a matrix with nonpositive off-diagonal elements, then the following statements are equivalent:

(i) $A$ is an M-matrix;

(ii) there exists a vector $\eta>0$ such that $A \eta>0$;

(iii) there exists a vector $\xi>0$ such that $\xi^{T} A>0$;

(iv) there exists a positive definite $n \times n$ diagonal matrix $D$ such that $A D+D A^{T}>0$. 
Lemma 2.9 [36] Let $A \geq 0$ be an $l \times l$ matrix with $\rho(A)<1$, then $\left(E_{l}-A\right)^{-1} \geq 0$, where $\rho(A)$ denotes the spectral radius of $A$ and $E_{l}$ is the identity matrix of size $l$.

Definition 2.5 Let $z^{*}=\left(x_{1}^{*}, x_{2}^{*}, \ldots, x_{n}^{*}, y_{1}^{*}, y_{2}^{*}, \ldots, y_{m}^{*}\right)^{T}$ be an equilibrium point of (1.1). If there exists a positive constant $\lambda$ with $-\lambda \in \mathcal{R}^{+}$such that for $t_{0} \in[-\vartheta, 0]_{\mathbb{T}}$, there exists $M>1$ such that for an arbitrary solution $z(t)=\left(x_{1}(t), x_{2}(t), \ldots, x_{n}(t), y_{1}(t), y_{2}(t), \ldots, y_{m}(t)\right)^{T}$ of $(1.1)$ with initial value $\phi(s)=\left(\varphi_{1}(s), \varphi_{2}(s), \ldots, \varphi_{n}(s), \psi_{1}(s), \psi_{2}(s), \ldots, \psi_{m}(s)\right)^{T}$ satisfies

$$
\left|z(t)-z^{*}\right|_{1} \leq M\left\|\phi-z^{*}\right\| e_{\ominus \lambda}\left(t, t_{0}\right), \quad t \in\left[t_{0}, \infty\right)_{\mathbb{T}}, t \geq t_{0},
$$

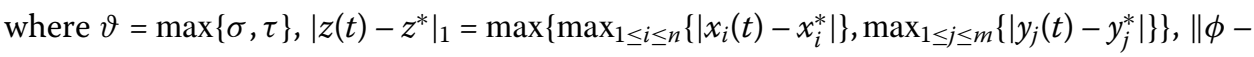
$z^{*} \|=\max \left\{\max _{1 \leq i \leq n} \max _{s \in[-\sigma, 0]_{\mathbb{T}}}\left\{\left|\varphi_{i}(s)-x_{i}^{*}\right|\right\}, \max _{1 \leq j \leq m} \max _{s \in[-\tau, 0]_{\mathbb{T}}}\left\{\left|\psi_{j}(s)-y_{j}^{*}\right|\right\}\right\}$. Then the equilibrium point $z^{*}$ is said to be globally exponentially stable.

\section{Existence and uniqueness of an equilibrium point}

In this section, we study the existence and uniqueness of an equilibrium point of (1.1).

Theorem 3.1 Let $(\mathrm{H})$ hold. Suppose further that $\rho(F)<1$, where $F=A^{-1} B L, A=\operatorname{diag}\left(a_{1}, a_{2}\right.$, $\left.\ldots, a_{n}, b_{1}, b_{2}, \ldots, b_{m}\right), L=\operatorname{diag}\left(L_{1}^{f}, L_{2}^{f}, \ldots, L_{m}^{f}, L_{1}^{g}, L_{2}^{g}, \ldots, L_{n}^{g}\right)$ and

$$
B=\left(\begin{array}{cc}
0_{n \times n} & P^{T} \\
Q^{T} & 0_{m \times m}
\end{array}\right),
$$

$P=\left(\left|c_{j i}\right|+\left|\alpha_{j i}\right|+\left|\beta_{j i}\right|\right)_{m \times n}, Q=\left(\left|d_{i j}\right|+\left|p_{i j}\right|+\left|q_{i j}\right|\right)_{n \times m}$. Then (1.1) has one unique equilibrium point.

Proof Let $z^{*}=\left(x_{1}^{*}, x_{2}^{*}, \ldots, x_{n}^{*}, y_{1}^{*}, y_{2}^{*}, \ldots, y_{m}^{*}\right)^{T}$ be an equilibrium point of (1.1), then we have

$$
\left\{\begin{aligned}
a_{i} x_{i}^{*}= & \sum_{j=1}^{m} c_{j i} f_{j}\left(y_{j}^{*}\right)+\bigwedge_{j=1}^{m} \alpha_{j i} f_{j}\left(y_{j}^{*}\right) \\
& +\bigwedge_{j=1}^{m} T_{j i} \mu_{j}+\bigvee_{j=1}^{m} \beta_{j i} f_{j}\left(y_{j}^{*}\right)+\bigvee_{j=1}^{m} H_{j i} \mu_{j}+I_{i}, \\
b_{j} y_{j}^{*}= & \sum_{i=1}^{n} d_{i j} g_{i}\left(x_{i}^{*}\right)+\bigwedge_{i=1}^{n} p_{i j} g_{i}\left(x_{i}^{*}\right)+\bigwedge_{i=1}^{n} F_{i j} v_{i} \\
& +\bigvee_{i=1}^{n} q_{i j} g_{i}\left(x_{i}^{*}\right)+\bigvee_{i=1}^{n} G_{i j} v_{i}+J_{j},
\end{aligned}\right.
$$

where $i=1,2, \ldots, n, j=1,2, \ldots, m$. Define a mapping $\Phi: \mathbb{R}^{n+m} \rightarrow \mathbb{R}^{n+m}$ as follows:

$$
\Phi\left(x_{1}, x_{2}, \ldots, x_{n}, y_{1}, y_{2}, \ldots, y_{m}\right)=\left(\Phi_{1}, \Phi_{2}, \ldots, \Phi_{n}, \Phi_{n+1}, \ldots, \Phi_{n+m}\right)^{T}
$$

where

$$
\left\{\begin{aligned}
\Phi_{i}= & a_{i}^{-1}\left[\sum_{j=1}^{m} c_{j i} f_{j}\left(y_{j}\right)+\bigwedge_{j=1}^{m} \alpha_{j j} f_{j}\left(y_{j}\right)\right. \\
& \left.+\bigwedge_{j=1}^{m} T_{j i} \mu_{j}+\bigvee_{j=1}^{m} \beta_{j i} f_{j}\left(y_{j}\right)+\bigvee_{j=1}^{m} H_{j i} \mu_{j}+I_{i}\right] \\
\Phi_{n+j}= & b_{j}^{-1}\left[\sum_{i=1}^{n} d_{i j} g_{i}\left(x_{i}\right)+\bigwedge_{i=1}^{n} p_{i j} g_{i}\left(x_{i}\right)+\bigwedge_{i=1}^{n} F_{i j} v_{i}\right. \\
& \left.+\bigvee_{i=1}^{n} q_{i j} g_{i}\left(x_{i}\right)+\bigvee_{i=1}^{n} G_{i j} v_{i}+J_{j}\right]
\end{aligned}\right.
$$

for $i=1,2, \ldots, n, j=1,2, \ldots, m$. Obviously, we need to show that $\Phi: \mathbb{R}^{n+m} \rightarrow \mathbb{R}^{n+m}$ is a contraction mapping on $\mathbb{R}^{n+m}$. In fact, for any $\Theta=\left(h_{1}, h_{2}, \ldots, h_{n}, v_{1}, v_{2}, \ldots, v_{m}\right)$ and $\bar{\Theta}=$ 


$$
\begin{aligned}
& \left(\bar{h}_{1}, \bar{h}_{2}, \ldots, \bar{h}_{n}, \bar{v}_{1}, \bar{v}_{2}, \ldots, \bar{v}_{m}\right) \in \mathbb{R}^{n+m}, \text { we have } \\
& \begin{aligned}
\left|\Phi_{i}\left(h_{i}\right)-\Phi_{i}\left(\bar{h}_{i}\right)\right| \\
=\left|a_{i}^{-1} \sum_{j=1}^{m} c_{j i}\left(f_{j}\left(v_{j}\right)-f_{j}\left(\bar{v}_{j}\right)\right)\right|+\left|a_{i}^{-1} \bigwedge_{j=1}^{m} \alpha_{j i}\left(f_{j}\left(v_{j}\right)-f_{j}\left(\bar{v}_{j}\right)\right)\right| \\
\\
+\left|a_{i}^{-1} \bigvee_{j=1}^{m} \beta_{j i}\left(f_{j}\left(v_{j}\right)-f_{j}\left(\bar{v}_{j}\right)\right)\right| \\
\leq a_{i}^{-1} \sum_{j=1}^{m}\left(\left|c_{j i}\right|+\left|\alpha_{j i}\right|+\left|\beta_{j i}\right|\right) L_{j}^{f}\left|v_{j}-\bar{v}_{j}\right|, \quad i=1,2, \ldots, n
\end{aligned}
\end{aligned}
$$

and

$$
\begin{aligned}
& \left|\Phi_{n+j}\left(v_{j}\right)-\Phi_{n+j}\left(\bar{v}_{j}\right)\right| \\
& =\left|b_{j}^{-1} \sum_{i=1}^{n} d_{i j}\left(g_{i}\left(h_{i}\right)-g_{i}\left(\bar{h}_{i}\right)\right)\right|+\left|b_{j}^{-1} \bigwedge_{i=1}^{n} p_{i j}\left(g_{i}\left(h_{i}\right)-g_{i}\left(\bar{h}_{i}\right)\right)\right| \\
& \quad+\left|b_{j}^{-1} \bigvee_{i=1}^{n} q_{i j}\left(g_{i}\left(h_{i}\right)-g_{i}\left(\bar{h}_{i}\right)\right)\right| \\
& \leq b_{j}^{-1} \sum_{i=1}^{n}\left(\left|d_{i j}\right|+\left|p_{i j}\right|+\left|q_{i j}\right|\right) L_{i}^{g}\left|h_{i}-\bar{h}_{i}\right|, \quad j=1,2, \ldots, m .
\end{aligned}
$$

It follows that

$$
\begin{aligned}
& \left|\Phi\left(h_{1}, h_{2}, \ldots, h_{n}, v_{1}, v_{2}, \ldots, v_{m}\right)-\Phi\left(\bar{h}_{1}, \bar{h}_{2}, \ldots, \bar{h}_{n}, \bar{v}_{1}, \bar{v}_{2}, \ldots, \bar{v}_{m}\right)\right| \\
& \quad \leq\left(\begin{array}{c}
a_{1}^{-1} \sum_{j=1}^{m}\left(\left|c_{j 1}\right|+\left|\alpha_{j 1}\right|+\left|\beta_{j 1}\right|\right) L_{j}^{f}\left|v_{j}-\bar{v}_{j}\right| \\
\vdots \\
a_{n}^{-1} \sum_{j=1}^{m}\left(\left|c_{j n}\right|+\left|\alpha_{j n}\right|+\left|\beta_{j n}\right|\right) L_{j}^{f}\left|v_{j}-\bar{v}_{j}\right| \\
b_{1}^{-1} \sum_{i=1}^{n}\left(\left|d_{i 1}\right|+\left|p_{i 1}\right|+\left|q_{i 1}\right|\right) L_{i}^{g}\left|h_{i}-\bar{h}_{i}\right| \\
\vdots \\
b_{m}^{-1} \sum_{i=1}^{n}\left(\left|d_{i m}\right|+\left|p_{i m}\right|+\left|q_{i m}\right|\right) L_{i}^{g}\left|h_{i}-\bar{h}_{i}\right|
\end{array}\right)=F\left(\begin{array}{c}
\left|h_{1}-\bar{h}_{1}\right| \\
\vdots \\
\left|h_{n}-\bar{h}_{n}\right| \\
\left|v_{1}-\bar{v}_{1}\right| \\
\vdots \\
\left|v_{m}-\bar{v}_{m}\right|
\end{array}\right) .
\end{aligned}
$$

Let $N$ be a positive integer. In view of (3.1), we have

$$
\begin{aligned}
& \left|\Phi^{N}\left(h_{1}, \ldots, h_{n}, v_{1}, \ldots, v_{m}\right)-\Phi^{N}\left(\bar{h}_{1}, \ldots, \bar{h}_{n}, \bar{v}_{1}, \ldots, \bar{v}_{m}\right)\right| \\
& \quad \leq F^{N}\left(\left|h_{1}-\bar{h}_{1}\right|, \ldots,\left|h_{n}-\bar{h}_{n}\right|,\left|v_{1}-\bar{v}_{1}\right|, \ldots,\left|v_{m}-\bar{v}_{m}\right|\right)^{T} .
\end{aligned}
$$

Since $\rho(F)<1$, we obtain $\lim _{N \rightarrow+\infty} F^{N}=0$, which implies that there exist a positive integer $M$ and a positive constant $r<1$ such that

$$
F^{M}=\left(A^{-1} B L\right)^{M}=\left(l_{i j}\right)^{(n+m) \times(n+m)}, \quad \sum_{j=1}^{n+m} l_{i j} \leq r, i=1,2, \ldots, n+m .
$$


Hence, we have

$$
\begin{array}{r}
\left|\Phi^{M}\left(h_{1}, \ldots, h_{n}, v_{1}, \ldots, v_{m}\right)-\Phi^{M}\left(\bar{h}_{1}, \ldots, \bar{h}_{n}, \bar{v}_{1}, \ldots, \bar{v}_{m}\right)\right| \\
\quad \leq F^{M}\left(\begin{array}{c}
\left|h_{1}-\bar{h}_{1}\right| \\
\vdots \\
\left|h_{n}-\bar{h}_{n}\right| \\
\left|v_{1}-\bar{v}_{1}\right| \\
\vdots \\
\left|v_{m}-\bar{v}_{m}\right|
\end{array}\right) \leq F^{M}\left(\begin{array}{c}
\left\|h_{1}-\bar{h}_{1}\right\| \\
\vdots \\
\left\|h_{n}-\bar{h}_{n}\right\| \\
\left\|v_{1}-\bar{v}_{1}\right\| \\
\vdots \\
\left\|v_{m}-\bar{v}_{m}\right\|
\end{array}\right)=\|\Theta-\bar{\Theta}\|\left(\begin{array}{c}
\sum_{j=1}^{n+m} l_{1 j} \\
\vdots \\
\sum_{j=1}^{n+m} l_{n j} \\
\sum_{j=1}^{n+m} l_{(n+1) j} \\
\vdots \\
\sum_{j=1}^{n+m} l_{(n+m) j}
\end{array}\right),
\end{array}
$$

which implies that $\left\|\Phi^{M}(\Theta)-\Phi^{M}(\bar{\Theta})\right\| \leq r\|\Theta-\bar{\Theta}\|$. Since $r<1$, it is obvious that the mapping $\Phi^{M}: \mathbb{R}^{n+m} \rightarrow \mathbb{R}^{n+m}$ is a contraction mapping. By the fixed point theorem of a Banach space, $\Phi$ possesses a unique fixed point in $\mathbb{R}^{n+m}$, that is, there exists a unique equilibrium point of (1.1). The proof of Theorem 3.1 is completed.

\section{Global exponential stability of an equilibrium point}

In this section, we study the global exponential stability of the equilibrium point of (1.1).

Theorem 4.1 Let $(\mathrm{H})$ and $\rho(F)<1$ hold. Suppose further that

$\left(\mathrm{H}_{5}\right) \max \left\{\epsilon_{1}, \epsilon_{2}\right\}<1$, where

$$
\epsilon_{1}=\max _{1 \leq i \leq n}\left\{a_{i} \delta_{i}^{+}+\left(\delta_{i}^{+}+a_{i}^{-1}\right) \sum_{j=1}^{m}\left(\left|c_{j i}\right|+\left|\alpha_{j i}\right|+\left|\beta_{j i}\right|\right) L_{j}^{f}\right\}
$$

and

$$
\epsilon_{2}=\max _{1 \leq j \leq m}\left\{b_{j} \eta_{j}^{+}+\left(\eta_{j}^{+}+b_{j}^{-1}\right) \sum_{i=1}^{n}\left(\left|d_{i j}\right|+\left|p_{i j}\right|+\left|q_{i j}\right|\right) L_{i}^{g}\right\} .
$$

Then the equilibrium point of (1.1) is globally exponentially stable.

Proof By Theorem 3.1, (1.1) has a unique equilibrium point $z^{*}=\left(x_{1}^{*}, x_{2}^{*}, \ldots, x_{n}^{*}, y_{1}^{*}, y_{2}^{*}, \ldots\right.$, $\left.y_{m}^{*}\right)^{T}$. Suppose that $z(t)=\left(x_{1}(t), x_{2}(t), \ldots, x_{n}(t), y_{1}(t), y_{2}(t), \ldots, y_{m}(t)\right)^{T}$ is an arbitrary solution of (1.1) with the initial condition $\phi(s)=\left(\varphi_{1}(s), \varphi_{2}(s), \ldots, \varphi_{n}(s), \psi_{1}(s), \psi_{2}(s), \ldots, \psi_{m}(s)\right)^{T}$. Let $w(t)=\left(u_{1}(t), u_{2}(t), \ldots, u_{n}(t), v_{1}(t), v_{2}(t), \ldots, v_{m}(t)\right)^{T}=z(t)-z^{*}$, where $u_{i}(t)=x_{i}(t)-x_{i}^{*}$, $v_{j}(t)=y_{j}(t)-y_{j}^{*}, i=1,2, \ldots, n, j=1,2, \ldots, m$. Then (1.1) can be rewritten as

$$
\left\{\begin{aligned}
u_{i}^{\Delta}(t)= & -a_{i} u_{i}\left(t-\delta_{i}(t)\right)+\sum_{j=1}^{m} c_{j i} \tilde{f}_{j}\left(v_{j}\left(t-\tau_{j i}(t)\right)\right)+\bigwedge_{j=1}^{m} \alpha_{j i} f_{j}\left(v_{j}\left(t-\tau_{j i}(t)\right)+y_{j}^{*}\right) \\
& -\bigwedge_{j=1}^{m} \alpha_{j i} f_{j}\left(y_{j}^{*}\right)+\bigvee_{j=1}^{m} \beta_{j i} f_{j}\left(v_{j}\left(t-\tau_{j i}(t)\right)+y_{j}^{*}\right) \\
& -\bigvee_{j=1}^{m} \beta_{j i} f_{j}\left(y_{j}^{*}\right), \quad i=1,2, \ldots, n, \\
v_{j}^{\Delta}(t)= & -b_{j} v_{j}\left(t-\eta_{j}(t)\right)+\sum_{i=1}^{n} d_{i j} \widetilde{g}_{i}\left(u_{i}\left(t-\sigma_{i j}(t)\right)\right)+\bigwedge_{i=1}^{n} p_{i j} g_{i}\left(u_{i}\left(t-\sigma_{i j}(t)\right)+x_{i}^{*}\right) \\
& -\bigwedge_{i=1}^{n} p_{i j} g_{i}\left(x_{i}^{*}\right)+\bigvee_{i=1}^{n} q_{i j} g_{i}\left(u_{i}\left(t-\sigma_{i j}(t)\right)+x_{i}^{*}\right) \\
& -\bigvee_{i=1}^{n} q_{i j} g_{i}\left(x_{i}^{*}\right), \quad j=1,2, \ldots, m
\end{aligned}\right.
$$

where $t \in \mathbb{T}$ and

$$
\tilde{f}_{j}\left(v_{j}\left(t-\tau_{j i}(t)\right)\right)=f_{j}\left(y_{j}\left(t-\tau_{j i}(t)\right)\right)-f_{j}\left(y_{j}^{*}\right), \quad \tilde{g}_{i}\left(u_{i}\left(t-\sigma_{i j}(t)\right)\right)=g_{i}\left(x_{i}\left(t-\sigma_{i j}(t)\right)\right)-g_{i}\left(x_{i}^{*}\right) .
$$


The initial condition of (4.1) is the following:

$$
\begin{cases}u_{i}(s)=\varphi_{i}(s)-x_{i}^{*}, & s \in[-\sigma, 0]_{\mathbb{T}}, i=1,2, \ldots, n, \\ v_{j}(s)=\psi_{j}(s)-y_{j}^{*}, & s \in[-\tau, 0]_{\mathbb{T}}, j=1,2, \ldots, m .\end{cases}
$$

We rewrite (4.1) as follows:

$$
\begin{aligned}
u_{i}^{\Delta}(s)+a_{i} u_{i}(s)= & a_{i} \int_{s-\delta_{i}(s)}^{s} u_{i}^{\Delta}(\theta) \Delta \theta+\sum_{j=1}^{m} c_{j i} \tilde{f}_{j}\left(v_{j}\left(s-\tau_{j i}(s)\right)\right)+\bigwedge_{j=1}^{m} \alpha_{j i} f_{j}\left(v_{j}\left(s-\tau_{j i}(s)\right)+y_{j}^{*}\right) \\
& -\bigwedge_{j=1}^{m} \alpha_{j i} f_{j}\left(y_{j}^{*}\right)+\bigvee_{j=1}^{m} \beta_{j i} f_{j}\left(v_{j}\left(s-\tau_{j i}(s)\right)+y_{j}^{*}\right) \\
& -\bigvee_{j=1}^{m} \beta_{j i} f_{j}\left(y_{j}^{*}\right), \quad i=1,2, \ldots, n
\end{aligned}
$$

and

$$
\begin{aligned}
v_{j}^{\Delta}(s)+b_{j} v_{j}(s)= & b_{j} \int_{s-\eta_{j}(s)}^{s} v_{j}^{\Delta}(\theta) \Delta \theta+\sum_{i=1}^{n} d_{i j} \widetilde{g}_{i}\left(u_{i}\left(s-\sigma_{i j}(s)\right)\right) \\
& +\bigwedge_{i=1}^{n} p_{i j} g_{i}\left(u_{i}\left(s-\sigma_{i j}(s)\right)+x_{i}^{*}\right) \\
& -\bigwedge_{i=1}^{n} p_{i j} g_{i}\left(x_{i}^{*}\right)+\bigvee_{i=1}^{n} q_{i j} g_{i}\left(u_{i}\left(s-\sigma_{i j}(s)\right)+x_{i}^{*}\right) \\
& -\bigvee_{i=1}^{n} q_{i j} g_{i}\left(x_{i}^{*}\right), \quad j=1,2, \ldots, m .
\end{aligned}
$$

Multiplying both sides of (4.2) by $e_{-a_{i}}(t, \sigma(s))$ and integrating on $\left[t_{0}, t\right]_{\mathbb{T}}$, where $t_{0} \in$ $[-\vartheta, 0]_{\mathbb{T}}$, we get

$$
\begin{aligned}
u_{i}(t)= & u_{i}\left(t_{0}\right) e_{-a_{i}}\left(t, t_{0}\right) \\
& +\int_{t_{0}}^{t} e_{-a_{i}}(t, \sigma(s))\left\{a_{i} \int_{s-\delta_{i}(s)}^{s} u_{i}^{\Delta}(\theta) \Delta \theta+\sum_{j=1}^{m} c_{j i} \widetilde{f}_{j}\left(v_{j}\left(s-\tau_{j i}(s)\right)\right)\right. \\
& +\bigwedge_{j=1}^{m} \alpha_{j i} f_{j}\left(v_{j}\left(s-\tau_{j i}(s)\right)+y_{j}^{*}\right)-\bigwedge_{j=1}^{m} \alpha_{j i} f_{j}\left(y_{j}^{*}\right)+\bigvee_{j=1}^{m} \beta_{j i} f_{j}\left(v_{j}\left(s-\tau_{j i}(s)\right)+y_{j}^{*}\right) \\
& \left.-\bigvee_{j=1}^{m} \beta_{j i} f_{j}\left(y_{j}^{*}\right)\right\} \Delta s, \quad i=1,2, \ldots, n .
\end{aligned}
$$

Similarly, multiplying both sides of $(4.3)$ by $e_{-b_{j}}(t, \sigma(s))$ and integrating on $\left[t_{0}, t\right]_{\mathbb{T}}$, we get

$$
\begin{aligned}
v_{j}(t)= & v_{j}\left(t_{0}\right) e_{-b_{j}}\left(t, t_{0}\right) \\
& +\int_{t_{0}}^{t} e_{-b_{j}}(t, \sigma(s))\left\{b_{j} \int_{s-\eta_{j}(s)}^{s} v_{j}^{\Delta}(\theta) \Delta \theta+\sum_{i=1}^{n} d_{i j} \widetilde{g}_{i}\left(u_{i}\left(s-\sigma_{i j}(s)\right)\right)\right.
\end{aligned}
$$




$$
\begin{aligned}
& +\bigwedge_{i=1}^{n} p_{i j} g_{i}\left(u_{i}\left(s-\sigma_{i j}(s)\right)+x_{i}^{*}\right)-\bigwedge_{i=1}^{n} p_{i j} g_{i}\left(x_{i}^{*}\right)+\bigvee_{i=1}^{n} q_{i j} g_{i}\left(u_{i}\left(s-\sigma_{i j}(s)\right)+x_{i}^{*}\right) \\
& \left.-\bigvee_{i=1}^{n} q_{i j} g_{i}\left(x_{i}^{*}\right)\right\} \Delta s, \quad j=1,2, \ldots, m .
\end{aligned}
$$

For a positive constant $\alpha<\min \left\{\min _{1 \leq i \leq n} a_{i}^{-}, \min _{1 \leq j \leq m} b_{j}^{-}\right\}$with $-\alpha \in \mathcal{R}^{+}$, we have $e_{\ominus \alpha}\left(t, t_{0}\right)>1$ for $t<t_{0}$. Take $M>\max \left\{\frac{1}{\epsilon_{1}}, \frac{1}{\epsilon_{2}}\right\}$. In view of $\left(\mathrm{H}_{5}\right)$, it is obvious that $M>1$. Hence, we have

$$
|w(t)|_{1} \leq M e_{\ominus \alpha}\left(t, t_{0}\right)\left\|\phi-z^{*}\right\|, \quad \forall t \in\left[-\vartheta, t_{0}\right]_{\mathbb{T}} .
$$

We claim that

$$
|w(t)|_{1} \leq M e_{\ominus \alpha}\left(t, t_{0}\right)\left\|\phi-z^{*}\right\|, \quad \forall t \in\left(t_{0},+\infty\right)_{\mathbb{T}}
$$

To prove this claim, we show that for any $p>1$, the following inequality holds:

$$
|w(t)|_{1}<p M e_{\ominus \alpha}\left(t, t_{0}\right)\left\|\phi-z^{*}\right\|, \quad \forall t \in\left(t_{0},+\infty\right)_{\mathbb{T}},
$$

which means that for $i=1,2, \ldots, n$, we have

$$
\left|u_{i}(t)\right|<p M e_{\ominus \alpha}\left(t, t_{0}\right)\left\|\phi-z^{*}\right\|, \quad \forall t \in\left(t_{0},+\infty\right)_{\mathbb{T}}
$$

and for $j=1,2, \ldots, m$, we have

$$
\left|v_{j}(t)\right|<p M e_{\ominus \alpha}\left(t, t_{0}\right)\left\|\phi-z^{*}\right\|, \quad \forall t \in\left(t_{0},+\infty\right)_{\mathbb{T}} .
$$

By way of contradiction, assume that (4.7) does not hold. Firstly, we consider the following two cases.

Case One: (4.8) is not true and (4.9) is true. Then there exist $t_{1} \in\left(t_{0},+\infty\right)_{\mathbb{T}}$ and $i_{0} \in$ $\{1,2, \ldots, n\}$ such that

$$
\begin{aligned}
& \left|u_{i_{0}}\left(t_{1}\right)\right| \geq p M e_{\ominus \alpha}\left(t_{1}, t_{0}\right)\left\|\phi-z^{*}\right\|, \quad\left|u_{i_{0}}(t)\right|<p M e_{\ominus \alpha}\left(t, t_{0}\right)\left\|\phi-z^{*}\right\|, \quad t \in\left(t_{0}, t_{1}\right)_{\mathbb{T}}, \\
& \left|u_{l}(t)\right|<p M e_{\ominus \alpha}\left(t, t_{0}\right)\left\|\phi-z^{*}\right\| \quad \text { for } l \neq i_{0}, t \in\left(t_{0}, t_{1}\right]_{\mathbb{T}}, l=1,2, \ldots, n .
\end{aligned}
$$

Hence, there must be a constant $c>1$ such that

$$
\begin{aligned}
& \left|u_{i_{0}}\left(t_{1}\right)\right|=c p M e_{\ominus \alpha}\left(t_{1}, t_{0}\right)\left\|\phi-z^{*}\right\|, \quad\left|u_{i_{0}}(t)\right|<c p M e_{\ominus \alpha}\left(t, t_{0}\right)\left\|\phi-z^{*}\right\|, \quad t \in\left(t_{0}, t_{1}\right)_{\mathbb{T}}, \\
& \left|u_{l}(t)\right|<c p M e_{\ominus \alpha}\left(t, t_{0}\right)\left\|\phi-z^{*}\right\| \quad \text { for } l \neq i_{0}, t \in\left(t_{0}, t_{1}\right]_{\mathbb{T}}, l=1,2, \ldots, n .
\end{aligned}
$$

Note that in view of (4.4), we have

$$
\begin{aligned}
\left|u_{i_{0}}\left(t_{1}\right)\right|= & \mid u_{i_{0}}\left(t_{0}\right) e_{-a_{i_{0}}}\left(t_{1}, t_{0}\right)+\int_{t_{0}}^{t_{1}} e_{-a_{i_{0}}}\left(t_{1}, \sigma(s)\right) \\
& \times\left\{a_{i_{0}} \int_{s-\delta_{i_{0}}(s)}^{s} u_{i_{0}}^{\Delta}(\theta) \Delta \theta+\sum_{j=1}^{m} c_{j i_{0}} \tilde{f}_{j}\left(v_{j}\left(s-\tau_{j i_{0}}(s)\right)\right)\right.
\end{aligned}
$$


Li et al. Advances in Difference Equations 2013, 2013:218

Page 10 of 16

http://www.advancesindifferenceequations.com/content/2013/1/218

$$
\begin{aligned}
& +\bigwedge_{j=1}^{m} \alpha_{j i_{0}} f_{j}\left(v_{j}\left(s-\tau_{j i_{0}}(s)\right)+y_{j}^{*}\right)-\bigwedge_{j=1}^{m} \alpha_{j i_{0}} f_{j}\left(y_{j}^{*}\right) \\
& \left.+\bigvee_{j=1}^{m} \beta_{j i_{0}} f_{j}\left(v_{j}\left(s-\tau_{j i_{0}}(s)\right)+y_{j}^{*}\right)-\bigvee_{j=1}^{m} \beta_{j i_{0}} f_{j}\left(y_{j}^{*}\right)\right\} \Delta s \mid \\
& =\mid u_{i_{0}}\left(t_{0}\right) e_{-a_{i_{0}}}\left(t_{1}, t_{0}\right)+\int_{t_{0}}^{t_{1}} e_{-a_{i_{0}}}\left(t_{1}, \sigma(s)\right) \\
& \times\left\{a _ { i _ { 0 } } \int _ { s - \delta _ { i _ { 0 } } ( s ) } ^ { s } \left[-a_{i_{0}} u_{i_{0}}\left(\theta-\delta_{i_{0}}(\theta)\right)+\sum_{j=1}^{m} c_{j i_{0}} \widetilde{f}_{j}\left(v_{j}\left(\theta-\tau_{j i_{0}}(\theta)\right)\right)\right.\right. \\
& +\bigwedge_{j=1}^{m} \alpha_{j i_{0}} f_{j}\left(v_{j}\left(\theta-\tau_{j i_{0}}(\theta)\right)+y_{j}^{*}\right)-\bigwedge_{j=1}^{m} \alpha_{j i_{0}} f_{j}\left(y_{j}^{*}\right) \\
& +\bigvee_{j=1}^{m} \beta_{j i_{0}} f_{j}\left(v_{j}\left(\theta-\tau_{j i_{0}}(\theta)\right)+y_{j}^{*}\right) \\
& \left.-\bigvee_{j=1}^{m} \beta_{j i_{0}} f_{j}\left(y_{j}^{*}\right)\right] \Delta \theta+\sum_{j=1}^{m} c_{j i_{0}} \widetilde{f}_{j}\left(v_{j}\left(s-\tau_{j i_{0}}(s)\right)\right) \\
& +\bigwedge_{j=1}^{m} \alpha_{j i_{0}} f_{j}\left(v_{j}\left(s-\tau_{j i_{0}}(s)\right)+y_{j}^{*}\right)-\bigwedge_{j=1}^{m} \alpha_{j i_{0}} f_{j}\left(y_{j}^{*}\right) \\
& \left.+\bigvee_{j=1}^{m} \beta_{j i_{0}} f_{j}\left(v_{j}\left(s-\tau_{j i_{0}}(s)\right)+y_{j}^{*}\right)-\bigvee_{j=1}^{m} \beta_{j i_{0}} f_{j}\left(y_{j}^{*}\right)\right\} \Delta s \mid \\
& \leq e_{-a_{i_{0}}}\left(t_{1}, t_{0}\right)\left\|\phi-z^{*}\right\|+\int_{t_{0}}^{t_{1}} e_{-a_{i_{0}}}\left(t_{1}, \sigma(s)\right) \\
& \times\left\{a _ { i _ { 0 } } \int _ { s - \delta _ { i _ { 0 } } ( s ) } ^ { s } \left[a_{i_{0}}\left|u_{i_{0}}\left(\theta-\delta_{i_{0}}(\theta)\right)\right|+\mid \sum_{j=1}^{m} c_{j i_{0}} \widetilde{f}_{j}\left(v_{j}\left(\theta-\tau_{j i_{0}}(\theta)\right)\right)\right.\right. \\
& +\bigwedge_{j=1}^{m} \alpha_{j i_{0}} f_{j}\left(v_{j}\left(\theta-\tau_{j i_{0}}(\theta)\right)+y_{j}^{*}\right)-\bigwedge_{j=1}^{m} \alpha_{j i_{0}} f_{j}\left(y_{j}^{*}\right)+\bigvee_{j=1}^{m} \beta_{j i_{0}} f_{j}\left(v_{j}\left(\theta-\tau_{j i_{0}}(\theta)\right)+y_{j}^{*}\right) \\
& \left.-\bigvee_{j=1}^{m} \beta_{j i_{0}} f_{j}\left(y_{j}^{*}\right) \mid\right] \Delta \theta+\mid \sum_{j=1}^{m} c_{j i_{0}} \widetilde{f}_{j}\left(v_{j}\left(s-\tau_{j i_{0}}(s)\right)\right) \\
& +\bigwedge_{j=1}^{m} \alpha_{j i i_{0}} f_{j}\left(v_{j}\left(s-\tau_{j i_{0}}(s)\right)+y_{j}^{*}\right)-\bigwedge_{j=1}^{m} \alpha_{j i_{0}} f_{j}\left(y_{j}^{*}\right) \\
& \left.+\bigvee_{j=1}^{m} \beta_{j i_{0}} f_{j}\left(v_{j}\left(s-\tau_{j i_{0}}(s)\right)+y_{j}^{*}\right)-\bigvee_{j=1}^{m} \beta_{j i_{0}} f_{j}\left(y_{j}^{*}\right) \mid\right\} \Delta s \\
& \leq\left\|\phi-z^{*}\right\|+\int_{t_{0}}^{t_{1}} e_{-a_{i_{0}}}\left(t_{1}, \sigma(s)\right)\left\{a _ { i _ { 0 } } \delta _ { i _ { 0 } } ^ { + } \left[a_{i_{0}} c p M e_{\ominus \alpha}\left(t_{1}, t_{0}\right)\left\|\phi-z^{*}\right\|\right.\right. \\
& \left.+\sum_{j=1}^{m}\left(\left|c_{j i_{0}}\right|+\left|\alpha_{j i_{0}}\right|+\left|\beta_{j i_{0}}\right|\right) L_{j}^{f} c p M e_{\ominus \alpha}\left(t_{1}, t_{0}\right)\left\|\phi-z^{*}\right\|\right] \\
& \left.+\sum_{j=1}^{m}\left(\left|c_{j i_{0}}\right|+\left|\alpha_{j i_{0}}\right|+\left|\beta_{j i_{0}}\right|\right) L_{j}^{f} c p M e_{\ominus \alpha}\left(t_{1}, t_{0}\right)\left\|\phi-z^{*}\right\|\right\} \Delta s
\end{aligned}
$$




$$
\begin{aligned}
& \leq\left\|\phi-z^{*}\right\| e_{\ominus \alpha}\left(t_{1}, t_{0}\right)+\int_{t_{0}}^{t_{1}} e_{-a_{i_{0}}}\left(t_{1}, \sigma(s)\right) \\
& \times\left\{a _ { i _ { 0 } } \delta _ { i _ { 0 } } ^ { + } \left[a_{i_{0}} c p M e_{\ominus \alpha}\left(t_{1}, t_{0}\right)\left\|\phi-z^{*}\right\|\right.\right. \\
& \left.+\sum_{j=1}^{m}\left(\left|c_{j i_{0}}\right|+\left|\alpha_{j i_{0}}\right|+\left|\beta_{j i_{0}}\right|\right) L_{j}^{f} c p M e_{\ominus \alpha}\left(t_{1}, t_{0}\right)\left\|\phi-z^{*}\right\|\right] \\
& \left.+\sum_{j=1}^{m}\left(\left|c_{j i_{0}}\right|+\left|\alpha_{j i_{0}}\right|+\left|\beta_{j i_{0}}\right|\right) L_{j}^{f} c p M e_{\ominus \alpha}\left(t_{1}, t_{0}\right)\left\|\phi-z^{*}\right\|\right\} \Delta s \\
& =\left\|\phi-z^{*}\right\| e_{\ominus \alpha}\left(t_{1}, t_{0}\right)+c p M e_{\ominus \alpha}\left(t_{1}, t_{0}\right)\left\|\phi-z^{*}\right\| \\
& \times \int_{t_{0}}^{t_{1}} e_{-a_{i_{0}}}\left(t_{1}, \sigma(s)\right)\left\{a_{i_{0}} \delta_{i_{0}}^{+}\left[a_{i_{0}}+\sum_{j=1}^{m}\left(\left|c_{j i_{0}}\right|+\left|\alpha_{j i_{0}}\right|+\left|\beta_{j i_{0}}\right|\right) L_{j}^{f}\right]\right. \\
& \left.+\sum_{j=1}^{m}\left(\left|c_{j i_{0}}\right|+\left|\alpha_{j i_{0}}\right|+\left|\beta_{j i_{0}}\right|\right) L_{j}^{f}\right\} \Delta s \\
& =\left\|\phi-z^{*}\right\| e_{\ominus \alpha}\left(t_{1}, t_{0}\right)-\frac{1}{a_{i_{0}}} c p M e_{\ominus \alpha}\left(t_{1}, t_{0}\right)\left\|\phi-z^{*}\right\| \\
& \times\left\{a_{i_{0}} \delta_{i_{0}}^{+}\left[a_{i_{0}}+\sum_{j=1}^{m}\left(\left|c_{j i_{0}}\right|+\left|\alpha_{j i_{0}}\right|+\left|\beta_{j i_{0}}\right|\right) L_{j}^{f}\right]\right. \\
& \left.+\sum_{j=1}^{m}\left(\left|c_{j i_{0}}\right|+\left|\alpha_{j i_{0}}\right|+\left|\beta_{j i_{0}}\right|\right) L_{j}^{f}\right\} \int_{t_{0}}^{t_{1}}\left(-a_{i_{0}}\right) e_{-a_{i_{0}}}\left(t_{1}, \sigma(s)\right) \Delta s \\
& =\left\|\phi-z^{*}\right\| e_{\ominus \alpha}\left(t_{1}, t_{0}\right)-\frac{1}{a_{i_{0}}}\left(e_{a_{i_{0}}}\left(t_{1}, t_{0}\right)-1\right) c p M e_{\ominus \alpha}\left(t_{1}, t_{0}\right)\left\|\phi-z^{*}\right\| \\
& \times\left\{a_{i_{0}} \delta_{i_{0}}^{+}\left[a_{i_{0}}+\sum_{j=1}^{m}\left(\left|c_{j i_{0}}\right|+\left|\alpha_{j i_{0}}\right|+\left|\beta_{j i_{0}}\right|\right) L_{j}^{f}\right]\right. \\
& \left.+\sum_{j=1}^{m}\left(\left|c_{j i_{0}}\right|+\left|\alpha_{j i_{0}}\right|+\left|\beta_{j i_{0}}\right|\right) L_{j}^{f}\right\} \\
& <c p M\left\|\phi-z^{*}\right\| e_{\ominus \alpha}\left(t_{1}, t_{0}\right) \\
& \times\left\{\frac{1}{c p M}+\frac{1}{a_{i_{0}}}\left[a_{i_{0}} \delta_{i_{0}}^{+}\left(a_{i_{0}}+\sum_{j=1}^{m}\left(\left|c_{j i_{0}}\right|+\left|\alpha_{j i_{0}}\right|+\left|\beta_{j i_{0}}\right|\right) L_{j}^{f}\right)\right.\right. \\
& \left.\left.+\sum_{j=1}^{m}\left(\left|c_{j i_{0}}\right|+\left|\alpha_{j i_{0}}\right|+\left|\beta_{j i_{0}}\right|\right) L_{j}^{f}\right]\right\} \\
& <c p M\left\|\phi-z^{*}\right\| e_{\ominus \alpha}\left(t_{1}, t_{0}\right) \\
& \times\left\{\frac{1}{M}+\frac{1}{a_{i_{0}}}\left[a_{i_{0}} \delta_{i_{0}}^{+}\left(a_{i_{0}}+\sum_{j=1}^{m}\left(\left|c_{j i_{0}}\right|+\left|\alpha_{j i_{0}}\right|+\left|\beta_{j i_{0}}\right|\right) L_{j}^{f}\right)\right.\right. \\
& \left.\left.+\sum_{j=1}^{m}\left(\left|c_{j i_{0}}\right|+\left|\alpha_{j i_{0}}\right|+\left|\beta_{j i_{0}}\right|\right) L_{j}^{f}\right]\right\}
\end{aligned}
$$


Li et al. Advances in Difference Equations 2013, 2013:218

Page 12 of 16

http://www.advancesindifferenceequations.com/content/2013/1/218

$$
\begin{aligned}
= & c p M\left\|\phi-z^{*}\right\| e_{\ominus \alpha}\left(t_{1}, t_{0}\right) \\
& \times\left(\frac{1}{M}+a_{i_{0}} \delta_{i_{0}}^{+}+\left(\delta_{i_{0}}^{+}+a_{i_{0}}^{-1}\right) \sum_{j=1}^{m}\left(\left|c_{j i_{0}}\right|+\left|\alpha_{j i_{0}}\right|+\left|\beta_{j i_{0}}\right|\right) L_{j}^{f}\right) \\
< & <p M e_{\ominus \alpha}\left(t_{1}, t_{0}\right)\left\|\phi-z^{*}\right\|,
\end{aligned}
$$

which is a contradiction.

Case Two: (4.8) is true and (4.9) is not true. Then there exist $t_{2} \in\left(t_{0},+\infty\right)_{\mathbb{T}}$ and $j_{0} \in$ $\{1,2, \ldots, n\}$ such that

$$
\begin{aligned}
& \left|v_{j_{0}}\left(t_{2}\right)\right| \geq p M e_{\ominus \alpha}\left(t_{2}, t_{0}\right)\left\|\phi-z^{*}\right\|, \quad\left|v_{j_{0}}(t)\right|<p M e_{\ominus \alpha}\left(t, t_{0}\right)\left\|\phi-z^{*}\right\|, \quad t \in\left(t_{0}, t_{2}\right)_{\mathbb{T}}, \\
& \left|v_{k}(t)\right|<p M e_{\ominus \alpha}\left(t, t_{0}\right)\left\|\phi-z^{*}\right\| \quad \text { for } k \neq j_{0}, t \in\left(t_{0}, t_{2}\right]_{\mathbb{T}}, k=1,2, \ldots, m .
\end{aligned}
$$

Hence, there must be a constant $c_{1}>1$ such that

$$
\begin{aligned}
& \left|v_{j_{0}}\left(t_{2}\right)\right|=c_{1} p M e_{\ominus \alpha}\left(t_{2}, t_{0}\right)\left\|\phi-z^{*}\right\|, \\
& \left|v_{j_{0}}(t)\right|<c_{1} p M e_{\ominus \alpha}\left(t, t_{0}\right)\left\|\phi-z^{*}\right\|, \quad t \in\left(t_{0}, t_{2}\right)_{\mathbb{T}}, \\
& \left|v_{k}(t)\right|<c_{1} p M e_{\ominus \alpha}\left(t, t_{0}\right)\left\|\phi-z^{*}\right\| \quad \text { for } k \neq j_{0}, t \in\left(t_{0}, t_{2}\right]_{\mathbb{T}}, k=1,2, \ldots, m .
\end{aligned}
$$

Note that in view of (4.5), we have

$$
\begin{aligned}
& \left|v_{j_{0}}\left(t_{2}\right)\right|=\mid v_{j_{0}}\left(t_{0}\right) e_{-b_{j_{0}}}\left(t_{2}, t_{0}\right)+\int_{t_{0}}^{t_{2}} e_{-b_{j_{0}}}\left(t_{2}, \sigma(s)\right) \\
& \times\left\{b _ { j _ { 0 } } \int _ { s - \eta _ { j _ { 0 } } ( s ) } ^ { s } \left[-b_{j_{0}} v_{j_{0}}\left(\theta-\eta_{j_{0}}(\theta)\right)+\sum_{i=1}^{n} d_{i j_{0}} \widetilde{g}_{i}\left(u_{i}\left(\theta-\sigma_{i j_{0}}(\theta)\right)\right)\right.\right. \\
& +\bigwedge_{i=1}^{n} p_{i j_{0}} g_{i}\left(u_{i}\left(\theta-\sigma_{i j_{0}}(\theta)\right)+x_{i}^{*}\right)-\bigwedge_{i=1}^{n} p_{i j_{0}} g_{i}\left(x_{i}^{*}\right) \\
& +\bigvee_{i=1}^{n} q_{i j_{0}} g_{i}\left(u_{i}\left(\theta-\sigma_{i j_{0}}(\theta)\right)+x_{i}^{*}\right) \\
& \left.-\bigvee_{i=1}^{n} q_{i j_{0}} g_{i}\left(x_{i}^{*}\right)\right] \Delta \theta+\sum_{i=1}^{n} d_{i j_{0}} \widetilde{g}_{i}\left(u_{i}\left(s-\sigma_{i j_{0}}(s)\right)\right) \\
& +\bigwedge_{i=1}^{n} p_{i j_{0}} g_{i}\left(u_{i}\left(s-\sigma_{i j_{0}}(s)\right)+x_{i}^{*}\right)-\bigwedge_{i=1}^{n} p_{i j_{0}} g_{i}\left(x_{i}^{*}\right) \\
& \left.+\bigvee_{i=1}^{n} q_{i j_{0}} g_{i}\left(u_{i}\left(s-\sigma_{i j_{0}}(s)\right)+x_{i}^{*}\right)-\bigvee_{i=1}^{n} q_{i j_{0}} g_{i}\left(x_{i}^{*}\right)\right\} \Delta s \\
& \leq e_{-b_{j}}\left(t_{2}, t_{0}\right)\left\|\phi-z^{*}\right\|+\mid \int_{t_{0}}^{t_{2}} e_{-b_{j}}\left(t_{2}, \sigma(s)\right) \\
& \times\left\{b _ { j _ { 0 } } \int _ { s - \eta _ { j _ { 0 } } ( s ) } ^ { s } \left[-b_{j_{0}} v_{j_{0}}\left(\theta-\eta_{j_{0}}(\theta)\right)+\sum_{i=1}^{n} d_{i j_{0}} \widetilde{g}_{i}\left(u_{i}\left(\theta-\sigma_{i j_{0}}(\theta)\right)\right)\right.\right. \\
& +\bigwedge_{i=1}^{n} p_{i j_{0}} g_{i}\left(u_{i}\left(\theta-\sigma_{i j_{0}}(\theta)\right)+x_{i}^{*}\right)-\bigwedge_{i=1}^{n} p_{i j_{0}} g_{i}\left(x_{i}^{*}\right)
\end{aligned}
$$




$$
\begin{aligned}
& +\bigvee_{i=1}^{n} q_{i j_{0}} g_{i}\left(u_{i}\left(\theta-\sigma_{i j_{0}}(\theta)\right)+x_{i}^{*}\right) \\
& \left.-\bigvee_{i=1}^{n} q_{i j o} g_{i}\left(x_{i}^{*}\right)\right] \Delta \theta+\sum_{i=1}^{n} d_{i j} \widetilde{\sigma}_{i}\left(u_{i}\left(s-\sigma_{i j_{0}}(s)\right)\right) \\
& +\bigwedge_{i=1}^{n} p_{i j} g_{i}\left(u_{i}\left(s-\sigma_{i j}(s)\right)+x_{i}^{*}\right)-\bigwedge_{i=1}^{n} p_{i j} g_{i}\left(x_{i}^{*}\right) \\
& \left.+\bigvee_{i=1}^{n} q_{i j} g_{i}\left(u_{i}\left(s-\sigma_{i j_{0}}(s)\right)+x_{i}^{*}\right)-\bigvee_{i=1}^{n} q_{i j_{0}} g_{i}\left(x_{i}^{*}\right)\right\} \Delta s \mid \\
& \leq\left\|\phi-z^{*}\right\|+\int_{t_{0}}^{t_{2}} e_{-b_{j 0}}\left(t_{2}, \sigma(s)\right)\left\{b _ { j _ { 0 } } \eta _ { j _ { 0 } } ^ { + } \left[b_{j_{0}} c_{1} p M e_{\ominus \alpha}\left(t_{2}, t_{0}\right)\left\|\phi-z^{*}\right\|\right.\right. \\
& \left.+\sum_{i=1}^{n}\left(\left|d_{i j_{0}}\right|+\left|p_{i j_{0}}\right|+\left|q_{i j_{0}}\right|\right) L_{i}^{g} c_{1} p M e_{\ominus \alpha}\left(t_{2}, t_{0}\right)\left\|\phi-z^{*}\right\|\right] \\
& \left.+\sum_{i=1}^{n}\left(\left|d_{i j_{0}}\right|+\left|p_{i j_{0}}\right|+\left|q_{i j_{0}}\right|\right) L_{i}^{g} c_{1} p M e_{\ominus \alpha}\left(t_{2}, t_{0}\right)\left\|\phi-z^{*}\right\|\right\} \Delta s \\
& \leq\left\|\phi-z^{*}\right\| e_{\ominus \alpha}\left(t_{2}, t_{0}\right)+\int_{t_{0}}^{t_{2}} e_{-b_{j_{0}}}\left(t_{2}, \sigma(s)\right) \\
& \times\left\{b _ { j _ { 0 } } \eta _ { j _ { 0 } } ^ { + } \left[b_{j_{0}} c_{1} p M e_{\ominus \alpha}\left(t_{2}, t_{0}\right)\left\|\phi-z^{*}\right\|\right.\right. \\
& \left.+\sum_{i=1}^{n}\left(\left|d_{i j_{0}}\right|+\left|p_{i j_{0}}\right|+\left|q_{i j_{0}}\right|\right) L_{i}^{g} c_{1} p M e_{\ominus \alpha}\left(t_{2}, t_{0}\right)\left\|\phi-z^{*}\right\|\right] \\
& \left.+\sum_{i=1}^{n}\left(\left|d_{i j_{0}}\right|+\left|p_{i j_{0}}\right|+\left|q_{i j_{0}}\right|\right) L_{i}^{g} c_{1} p M e_{\ominus \alpha}\left(t_{2}, t_{0}\right)\left\|\phi-z^{*}\right\|\right\} \Delta s \\
& =\left\|\phi-z^{*}\right\| e_{\ominus \alpha}\left(t_{2}, t_{0}\right)+c_{1} p M e_{\ominus \alpha}\left(t_{2}, t_{0}\right)\left\|\phi-z^{*}\right\| \\
& \times \int_{t_{0}}^{t_{2}} e_{-b_{j_{0}}}\left(t_{2}, \sigma(s)\right)\left\{b_{j_{0}} \eta_{j_{0}}^{+}\left[b_{j_{0}}+\sum_{i=1}^{n}\left(\left|d_{i j_{0}}\right|+\left|p_{i j_{0}}\right|+\left|q_{i j_{0}}\right|\right) L_{i}^{g}\right]\right. \\
& \left.+\sum_{i=1}^{n}\left(\left|d_{i j_{0}}\right|+\left|p_{i j_{0}}\right|+\left|q_{i j_{0}}\right|\right) L_{i}^{g}\right\} \Delta s \\
& =\left\|\phi-z^{*}\right\| e_{\ominus \alpha}\left(t_{2}, t_{0}\right)-\frac{1}{b_{j_{0}}} c_{1} p M e_{\ominus \alpha}\left(t_{2}, t_{0}\right)\left\|\phi-z^{*}\right\| \\
& \times\left\{b_{j_{0}} \eta_{j_{0}}^{+}\left[b_{j_{0}}+\sum_{i=1}^{n}\left(\left|d_{i j_{0}}\right|+\left|p_{i j_{0}}\right|+\left|q_{i j_{0}}\right|\right) L_{i}^{g}\right]\right. \\
& \left.+\sum_{i=1}^{n}\left(\left|d_{i j_{0}}\right|+\left|p_{i j_{0}}\right|+\left|q_{i j_{0}}\right|\right) L_{i}^{g}\right\} \int_{t_{0}}^{t_{2}} e_{-b_{j_{0}}}\left(t_{2}, \sigma(s)\right) \Delta s \\
& =\left\|\phi-z^{*}\right\| e_{\ominus \alpha}\left(t_{2}, t_{0}\right)-\frac{1}{b_{j_{0}}}\left(e_{b_{j_{0}}}\left(t_{2}, t_{0}\right)-1\right) c_{1} p M e_{\ominus \alpha}\left(t_{2}, t_{0}\right)\left\|\phi-z^{*}\right\| \\
& \times\left\{b_{j_{0}} \eta_{j_{0}}^{+}\left[b_{j_{0}}+\sum_{i=1}^{n}\left(\left|d_{i j_{0}}\right|+\left|p_{i j_{0}}\right|+\left|q_{i j_{0}}\right|\right) L_{i}^{g}\right]\right.
\end{aligned}
$$




$$
\begin{aligned}
& \left.+\sum_{i=1}^{n}\left(\left|d_{i j_{0}}\right|+\left|p_{i j_{0}}\right|+\left|q_{i j_{0}}\right|\right) L_{i}^{g}\right\} \\
< & c_{1} p M\left\|\phi-z^{*}\right\| e_{\ominus \alpha}\left(t_{2}, t_{0}\right) \\
& \times\left\{\frac{1}{c_{1} p M}+\frac{1}{b_{j 0}}\left[b_{j_{0}} \eta_{j_{0}}^{+}\left(b_{j_{0}}+\sum_{i=1}^{n}\left(\left|d_{i j_{0}}\right|+\left|p_{i j_{0}}\right|+\left|q_{i j_{0}}\right|\right) L_{i}^{g}\right)\right.\right. \\
& \left.\left.+\sum_{i=1}^{n}\left(\left|d_{i j_{0}}\right|+\left|p_{i j_{0}}\right|+\left|q_{i j_{0}}\right|\right) L_{i}^{g}\right]\right\} \\
< & c_{1} p M\left\|\phi-z^{*}\right\| e_{\ominus \alpha}\left(t_{2}, t_{0}\right) \\
& \times\left\{\frac{1}{M}+\frac{1}{b_{j_{0}}}\left[b_{j_{0}} \eta_{j_{0}}^{+}\left(b_{j_{0}}+\sum_{i=1}^{n}\left(\left|d_{i j_{0}}\right|+\left|p_{i j_{0}}\right|+\left|q_{i j_{0}}\right|\right) L_{i}^{g}\right)\right.\right. \\
& \left.\left.+\sum_{i=1}^{n}\left(\left|d_{i j_{0}}\right|+\left|p_{i j_{0}}\right|+\left|q_{i j_{0}}\right|\right) L_{i}^{g}\right]\right\} \\
< & \left.c_{1} p M\left\|\phi-z^{*}\right\| e_{\ominus \alpha}\left(t_{2}, t_{0}\right)\left(\frac{1}{M}+b_{j} \eta_{j}^{+}+\left(t_{2}, t_{0}\right) \| \phi-z_{j}^{-1}\right) \sum_{i=1}^{n}\left(\left|d_{i j}\right|+\left|p_{i j}\right|+\left|q_{i j}\right|\right) L_{i}^{g}\right)
\end{aligned}
$$

which is also a contradiction.

By the above two cases, for other cases of negative proposition of (4.7), we can obtain a contradiction. Therefore, (4.7) holds. Let $p \rightarrow 1$, then (4.6) holds. Hence, we have that

$$
|w(t)|_{1} \leq M\left\|\phi-z^{*}\right\| e_{\ominus \alpha}\left(t, t_{0}\right), \quad t \in[-\vartheta, \infty)_{\mathbb{T}}, t \geq t_{0}
$$

which means that the equilibrium point $z^{*}$ of (1.1) is globally exponentially stable. The proof of Theorem 4.1 is completed.

\section{Example}

In this section, we present an example to illustrate the feasibility of our results.

Example 5.1 Let $n=m=2$. Consider the following fuzzy BAM system with delays in leakage terms on a time scale $\mathbb{T}$ :

$$
\left\{\begin{aligned}
x_{i}^{\Delta}(t)= & -a_{i} x_{i}\left(t-\delta_{i}(t)\right)+\sum_{j=1}^{2} c_{j i} f_{j}\left(y_{j}\left(t-\tau_{j i}(t)\right)\right)+\bigwedge_{j=1}^{2} \alpha_{j i} f_{j}\left(y_{j}\left(t-\tau_{j i}(t)\right)\right) \\
& +\bigwedge_{j=1}^{2} T_{j i} \mu_{j}+\bigvee_{j=1}^{2} \beta_{j i} f_{j}\left(y_{j}\left(t-\tau_{j i}(t)\right)\right)+\bigvee_{j=1}^{2} H_{j i} \mu_{j}+I_{i}, \quad t \in \mathbb{T}, i=1,2, \\
y_{j}^{\Delta}(t)= & -b_{j} y_{j}\left(t-\eta_{j}(t)\right)+\sum_{i=1}^{2} d_{i j} g_{i}\left(x_{i}\left(t-\sigma_{i j}(t)\right)\right)+\bigwedge_{i=1}^{2} p_{i j} g_{i}\left(x_{i}\left(t-\sigma_{i j}(t)\right)\right) \\
& +\bigwedge_{i=1}^{2} F_{i j} v_{i}+\bigvee_{i=1}^{2} q_{i j} g_{i}\left(x_{i}\left(t-\sigma_{i j}(t)\right)\right)+\bigvee_{i=1}^{2} G_{i j} v_{i}+J_{j}, \quad t \in \mathbb{T}, j=1,2,
\end{aligned}\right.
$$

where time delays $\delta_{i}, \tau_{j i}, \eta_{j}, \sigma_{i j}, i, j=1,2$ are defined as those in system (1.1) and the coefficients are as follows:

$$
\begin{array}{ll}
\left(\begin{array}{cc}
a_{1} & 0 \\
0 & a_{2}
\end{array}\right)=\left(\begin{array}{ll}
3 & 0 \\
0 & 4
\end{array}\right), \quad\left(\begin{array}{cc}
b_{1} & 0 \\
0 & b_{2}
\end{array}\right)=\left(\begin{array}{cc}
2 & 0 \\
0 & 3
\end{array}\right), \\
\left(c_{j i}\right)_{2 \times 2}=\left(\begin{array}{ll}
0.01 & 0.03 \\
0.06 & 0.04
\end{array}\right), \quad\left(\alpha_{j i}\right)_{2 \times 2}=\left(\begin{array}{cc}
0.02 & 0 \\
-0.01 & 0.04
\end{array}\right),
\end{array}
$$




$$
\begin{aligned}
& \left(\beta_{j i}\right)_{2 \times 2}=\left(\begin{array}{cc}
0.05 & -0.03 \\
0 & 0.02
\end{array}\right), \quad\left(d_{i j}\right)_{2 \times 2}=\left(\begin{array}{ll}
0.03 & 0.01 \\
0.04 & 0.02
\end{array}\right), \\
& \left(p_{i j}\right)_{2 \times 2}=\left(\begin{array}{cc}
0.03 & -0.02 \\
0.01 & 0.04
\end{array}\right), \quad\left(q_{i j}\right)_{2 \times 2}=\left(\begin{array}{cc}
0 & -0.01 \\
-0.02 & 0.03
\end{array}\right), \\
& \left(\delta_{i}(t)\right)_{2 \times 2}=\left(\begin{array}{cc}
0.001|\sin t| & 0 \\
0 & 0.006|\cos t|
\end{array}\right), \quad\left(I_{i}\right)_{2 \times 1}=\left(\begin{array}{l}
3 \\
4
\end{array}\right), \\
& \left(\eta_{j}(t)\right)_{2 \times 2}=\left(\begin{array}{cc}
0.002|\sin 2 t| & 0 \\
0 & 0.004|\sin t-1|
\end{array}\right), \\
& \left(J_{j}\right)_{2 \times 1}=\left(\begin{array}{l}
2 \\
1
\end{array}\right), \quad f_{j}\left(u_{j}\right)=0.02\left|u_{j}\right|, \\
& g_{i}\left(v_{i}\right)=0.04\left(\left|v_{i}+1\right|-\left|v_{i}-1\right|\right), \quad \mu_{j}=v_{i}=1, \quad i, j=1,2,
\end{aligned}
$$

and $\left(T_{j i}\right)_{2 \times 2}=\left(H_{j i}\right)_{2 \times 2}=\left(F_{i j}\right)_{2 \times 2}=\left(G_{i j}\right)_{2 \times 2}$ are identity matrices. By calculating, we have $L_{j}^{f}=L_{i}^{g}=0.02$. We can verify that for $\mathbb{T}=\mathbb{R}$ and $\mathbb{T}=\mathbb{Z}$, all the conditions of Theorem 3.1 and Theorem 4.1 are satisfied. Hence, for $\mathbb{T}=\mathbb{R}$ or $\mathbb{T}=\mathbb{Z}$, (5.1) always has one unique equilibrium point, which is globally exponentially stable.

\section{Competing interests}

The authors declare that they have no competing interests.

\section{Authors' contributions}

All authors typed, read and approved the final manuscript.

\section{Acknowledgements}

This work is supported by the National Natural Sciences Foundation of People's Republic of China under Grant 10971183.

\section{Received: 25 April 2013 Accepted: 9 July 2013 Published: 22 July 2013}

\section{References}

1. Kosko, B: Bidirectional associative memories. IEEE Trans. Syst. Man Cybern. 18, 49-60 (1988)

2. Zhao, HY: Global stability of bidirectional associative memory neural networks with distributed delays. Phys. Lett. A 297, 182-190 (2002)

3. Li, YK: Existence and stability of periodic solution for BAM neural networks with distributed delays. Appl. Math. Comput. 159, 847-862 (2004)

4. Park, JH, Park, CH, Kwon, OM, Lee, SM: A new stability criterion for bidirectional associative memory neural networks of neutral-type. Appl. Math. Comput. 199, 716-722 (2008)

5. Zhou, Q: Global exponential stability of BAM neural networks with distributed delays and impulses. Nonlinear Anal., Real World Appl. 10, 144-153 (2009)

6. Li, YK: Global exponential stability of BAM neural networks with delays and impulses. Chaos Solitons Fractals 24, 279-285 (2005)

7. Zheng, B, Zhang, Y, Zhang, C: Global existence of periodic solutions on a simplified BAM neural network model with delays. Chaos Solitons Fractals 37, 1397-1408 (2008)

8. Xia, Y, Cao, JD, Lin, M: New results on the existence and uniqueness of almost periodic solution for BAM neural networks with continuously distributed delays. Chaos Solitons Fractals 31, 928-936 (2007)

9. Zhang, L, Si, L: Existence and exponential stability of almost periodic solution for BAM neural networks with variable coefficients and delays. Appl. Math. Comput. 194, 215-223 (2007)

10. Liu, C, Li, C, Liao, X: Variable-time impulses in BAM neural networks with delays. Neurocomputing 74, 3286-3295 (2011)

11. Yang, T, Yang, L, Wu, C, Chua, L: Fuzzy cellular neural networks: theory. In: Proceedings of IEEE International Workshop on Cellular Neural Networks and Applications, pp. 181-186 (1996)

12. Yuan, K, Cao, JD, Deng, J: Exponential stability and periodic solutions of fuzzy cellular neural networks with time-varying delays. Neurocomputing 69, 1619-1627 (2006)

13. Zhang, Q, Xiang, R: Global asymptotic stability of fuzzy cellular neural networks with time-varying delays. Phys. Lett. A 372, 3971-3977 (2008)

14. Song, Q, Cao, JD: Dynamical behaviors of discrete-time fuzzy cellular neural networks with variable delays and impulses. J. Franklin Inst. 345, 39-59 (2008) 
15. Wang, J, Lu, J: Global exponential stability of fuzzy cellular neural networks with delays and reaction-diffusion terms. Chaos Solitons Fractals 38, 878-885 (2008)

16. Li, YK, Yang, L, Wu, WQ: Periodic solutions for a class of fuzzy BAM neural networks with distributed delays and variable coefficients. Int. J. Bifurc. Chaos 20, 1551-1565 (2010)

17. Liu, Y, Tang, W: Exponential stability of fuzzy cellular neural networks with constant and time-varying delays. Phys. Lett. A 323, 224-233 (2004)

18. Gopalsamy, K: Stability and Oscillations in Delay Differential Equations of Population Dynamics. Kluwer Academic, Dordrecht (1992)

19. Li, X, Cao, JD: Delay-dependent stability of neural networks of neutral type with time delay in the leakage term. Nonlinearity 23, 1709-1726 (2010)

20. Li, X, Rakkiyappan, R, Balasubramanian, P: Existence and global stability analysis of equilibrium of fuzzy cellular neural networks with time delay in the leakage term under impulsive perturbations. J. Franklin Inst. 348, 135-155 (2011)

21. Li, X, Fu, X, Balasubramaniam, P, Rakkiyappan, R: Existence, uniqueness and stability analysis of recurrent neural networks with time delay in the leakage term under impulsive perturbations. Nonlinear Anal., Real World Appl. 11, 4092-4108 (2010)

22. Balasubramaniam, P, Vembarasan, V, Rakkiyappan, R: Leakage delays in T-S fuzzy cellular neural networks. Neural Process. Lett. 33, 111-136 (2011)

23. Balasubramaniam, P, Kalpana, M, Rakkiyappan, R: Global asymptotic stability of BAM fuzzy cellular neural networks with time delays in the leakage term, discrete and unbounded distributed delays. Math. Comput. Model. 53, 839-853 (2011)

24. Liu, BW: Global exponential stability for BAM neural networks with time-varying delays in the leakage terms. Nonlinear Anal., Real World Appl. 14, 559-566 (2013)

25. Hilger, S: Analysis on measure chains - a unified approach to continuous and discrete calculus. Results Math. 18 18-56 (1990)

26. Zhang, Z, Liu, K: Existence and global exponential stability of a periodic solution to interval general bidirectional associative memory (BAM) neural networks with multiple delays on time scales. Neural Netw. 24, 427-439 (2011)

27. Li, YK, Chen, XR, Zhao, L: Stability and existence of periodic solutions to delayed Cohen-Grossberg BAM neural networks with impulses on time scales. Neurocomputing 72, 1621-1630 (2009)

28. Li, YK, Gao, S: Global exponential stability for impulsive BAM neural networks with distributed delays on time scales. Neural Process. Lett. 31, 65-91 (2010)

29. Zhang, JM, Fan, M, Zhu, HP: Existence and roughness of exponential dichotomies of linear dynamic equations on time scales. Comput. Math. Appl. 59, 2658-2675 (2010)

30. Lakshmikantham, V, Vatsala, AS: Hybrid systems on time scales. J. Comput. Appl. Math. 141, 227-235 (2002)

31. Li, YK, Zhao, KH: Robust stability of delayed reaction-diffusion recurrent neural networks with Dirichlet boundary conditions on time scales. Neurocomputing 74, 1632-1637 (2011)

32. Li, YK, Zhang, TW: Global exponential stability of fuzzy interval delayed neural networks with impulses on time scales. Int. J. Neural Syst. 19, 449-456 (2009)

33. Li, YK, Zhao, KH, Ye, Y: Stability of reaction-diffusion recurrent neural networks with distributed delays and Neumann boundary conditions on time scales. Neural Process. Lett. 36, 217-234 (2012)

34. Li, YK, Wang, C: Uniformly almost periodic functions and almost periodic solutions to dynamic equations on time scales. Abstr. Appl. Anal. 2011, Article ID 341520 (2011)

35. Bohner, M, Peterson, A: Dynamic Equations on Time Scales: An Introduction with Applications. Birkhäuser, Boston (2001)

36. Berman, A, Plemmons, R: Nonnegative Matrices in the Mathematical Science. Academic Press, New York (1979)

doi:10.1186/1687-1847-2013-218

Cite this article as: Li et al.: Existence and exponential stability of an equilibrium point for fuzzy BAM neural

networks with time-varying delays in leakage terms on time scales. Advances in Difference Equations 2013 2013:218.

\section{Submit your manuscript to a SpringerOpen ${ }^{\circ}$ journal and benefit from:}

- Convenient online submission

- Rigorous peer review

- Immediate publication on acceptance

- Open access: articles freely available online

- High visibility within the field

- Retaining the copyright to your article 